\title{
Das Spannungsfeld von Transparenz und Geheimhaltung im demokratischen Staat
}

\section{Elke Gurlit}

Am 11. Oktober 1987 wurde Uwe Barschel, der damalige Ministerpräsident des Landes Schleswig-Holstein, tot in der Badewanne eines Hotelzimmers in Genf aufgefunden. Seinem Tod war eine politische Affäre vorausgegangen, in deren Verlauf Barschel, der Bespitzelung eines politischen Konkurrenten verdächtigt, seine Unschuld mit einem „Ehrenwort“ beteuert hatte. Die These, ein Mann habe auf den befürchteten Verlust seiner bürgerlichen Ehre nur im Suizid einen Ausweg gesehen, war von Beginn an umstritten. Ermittlungen wurden nachlässig geführt, Beweismittel nicht sorgfältig dokumentiert. Barschel hatte Insiderkenntnisse von anrüchigen Geschäften, etwa der Iran-Contra-Affäre und dem illegalen Verkauf von U-Boot-Zeichnungen an das südafrikanische Apartheid-Regime. Ein später die Ermittlungen führender Staatsanwalt ist überzeugt, dass Barschel ermordet wurde. ${ }^{1}$ Seine Ermittlungen wurden behindert, die Mord-These lebt weiter. Die Barschel-Affäre ist nicht nur einer der größten politischen Skandale der Bundesrepublik Deutschland, sondern auch eines ihrer größten Geheimnisse.

Geheimnisse gehören zu den Narrativen vieler Staaten. Sie sind indes nicht nur Stoff für Legenden und Verschwörungstheorien, sondern auch Quelle für Bedrohungsgefühle und Ängste. Als Gegenbild zum arkanen Staat erscheint der transparente Staat, der sein Wissen mit den Bürgern teilt. Indessen liegt auf der Hand, dass ein geheimnisloser Staat nicht vorstellbar, auch nicht wünschbar ist, weil politische Gestaltung ebenso wie ihre administrative Umsetzung auf Freiräume angewiesen sind. Transparenz und Geheimhaltung bilden deshalb ein Spannungsfeld. Ein Medium für die Zuordnung von Publizitäts- und Geheimhaltungssphären ist das Recht.

1 Wille, Ein Mord, der keiner sein durfte, 2011. 


\section{A. Verfassungsrechtliche Determinanten und legislative Spielräume}

\section{Die Verfassung als Rahmen}

Das Grundgesetz ist eine Rahmenordnung, innerhalb deren sich Gesetzgebung, Rechtsprechung und Verwaltung entfalten. ${ }^{2}$ Dies schließt zwar nicht aus, dass die Verfassung auch strikte Handlungsaufträge enthält; einen Gesetzgebungsauftrag zur Schaffung eines allgemeinen Informationszugangsrechts der Bürger gegenüber Verwaltung und Regierung enthält das Grundgesetz - anders als die südafrikanische Verfassung ${ }^{3}$ - hingegen nicht, und ein derartiges Recht besteht auch nicht verfassungsunmittelbar als Grundrecht. ${ }^{4}$ Allerdings ist die grundlegende Transparenz staatlichen Handelns Ausfluss des demokratischen Prinzips. Zudem fordert das Rechtsstaatsprinzip die Voraussehbarkeit und Berechenbarkeit staatlichen Handelns und stellt damit ebenfalls Publizitätsanforderungen. Demokratieund Rechtsstaatsprinzip sind als Staatsstrukturprinzipien tragende Säulen der Verfassung, ohne indessen das gebotene Handeln des Gesetzgebers detailliert vorzuzeichnen. ${ }^{5}$

Orientierungen erhält der Gesetzgeber allerdings auch durch verfassungsrechtliche Anforderungen an die Geheimhaltung. Diese beziehen sich sowohl auf geschützte öffentliche Belange als auch auf private Geheimhaltungsinteressen. So ist insbesondere ein unausforschbarer Kernbe-

2 Zum Verständnis der Verfassung als Rahmenordnung grundlegend Ernst-Wolfgang Böckenförde, Die Methoden der Verfassungsinterpretation, NJW 1976, S. 2089.

3 Section 32 (2) Constitution of the Republic of South Africa.

4 So auch im Ergebnis Sonja Wirtz/Stefan Brink, Die verfassungsrechtliche Verankerung der Informationszugangsfreiheit, NVwZ 2015, S. 1166. Das Grundrecht der Informationsfreiheit nach Art. 5 Abs. 1 S. 1 GG gewährt Zugang zu allgemein zugänglichen Informationsquellen. Amtliche Informationen sind allgemein zugänglich, wenn dies gesetzlich vorgesehen ist, BVerfGE 103, S. 44 (61); zur grundrechtsdogmatisch verfehlten Schutzbereichsbestimmung Elke Gurlit, Konturen eines Informationsverwaltungsrechts, DVB1. 2003, S. 1121; s.a. Friedrich Schoch, IFG-Kommentar, 2009, Einleitung Rn. 153. Aus der Pressefreiheit nach Art. 5 Abs. 1 S. 2 GG soll ein verfassungsunmittelbarer Auskunftsanspruch der Presse folgen, BVerwGE 146, S. 56.

5 Weitergehend Arno Scherzberg, Die Öffentlichkeit der Verwaltung, 2000, S. 291 ff., der von einem demokratischen Verteilungsprinzip ausgeht, demzufolge eine Regelvermutung für die freie Zugänglichkeit staatlicher Informationen besteht. 
reich exekutiver Eigenverantwortung der Regierung anerkannt. ${ }^{6}$ Dem grundrechtlichen Schutz der Berufsfreiheit und der Eigentumsgarantie unterfallen schutzwürdige Betriebs- und Geschäftsgeheimnisse, ${ }^{7}$ die allerdings zugunsten legitimer Gemeinwohlbelange eingeschränkt werden können. Engere Fesseln werden dem Gesetzgeber für Beschränkungen des grundrechtlich gewährleisteten personenbezogenen Datenschutzes angelegt. $^{8}$

\section{Informationszugangsgesetzgebung als Akt rechtspolitischer Gestaltung}

Die Schaffung von Transparenz und von Informationsrechten ist nach alledem in wesentlichen Teilen Rechtspolitik, die durch die Verfassung Orientierungen, aber keine strikten Handlungsaufträge erhält. ${ }^{9}$ Der Bundesgesetzgeber bedurfte hierzu allerdings einiger Anstöße. So verpflichtete ihn in den 90er Jahren des vergangenen Jahrhunderts die europäische Umweltinformationsrichtlinie, ein voraussetzungsloses Informationszugangsrecht der Bürger zu Umweltinformationen der Behörden zu schaffen. ${ }^{10}$ Nachdem einige Bundesländer durch allgemeine Informationsfreiheitsgesetze die Zugangsrechte der Bürger zu den Informationsbeständen der Landesund Kommunalbehörden über den engeren Rahmen der Umweltinformationen hinaus geöffnet hatten, folgte der Bund den drängender werdenden Rufen ${ }^{11}$ nach einem allgemeinen Informationsfreiheitsgesetz des Bundes.

6 BVerfGE 67, S. 100, 139; 110, S. 199, 216; 124, S. 78 jeweils im Verhältnis zu parlamentarischen Untersuchungsausschüssen.

7 BVerfGE 115, S. 205, 229, 248 zum Schutz nach Art. 12 Abs. 1 GG; zum Schutz als Eigentum BVerwG BeckRS 2014, 45857, Rn. 8.

8 BVerfGE 65, S. 1, 43 f. für das informationelle Selbstbestimmungsrecht; dies gilt gleichermaßen für das Telekommunikationsgeheimnis und die Unverletzlichkeit der Wohnung, dazu Elke Gurlit, Verfassungsrechtliche Rahmenbedingungen des Datenschutzes, NJW 2010, S. 1035.

9 So auch die Einschätzung von Schoch, Fn. 4, Einl. Rn 47.

10 Richtlinie 90/313/EWG vom 7. Juni 1990 über den freien Zugang zu Informationen über die Umwelt, ABl.EG L 158/56; nunmehr Richtlinie 2003/4/EG vom 28. Januar 2003, AB1.EU 2003.

11 Einflussreich war der Professorenentwurf von Friedrich Schoch/Michael Kloepfer, Informationsfreiheitsgesetz (IFG-ProfE), 2002; s.a. die Habilitationsschriften von Scherzberg, Fn. 5; Dieter Kugelmann, Die informatorische Rechtsstellung des Bürgers, 2001; Andreas Fisahn, Demokratie und Öffentlichkeitsbeteiligung, 2002; Matthias Rossi, Informationsfreiheit und Verfassungsrecht, 2004; Bernhard Wege- 
Das zum 1. Januar 2006 in Kraft getretene Gesetz zur Regelung des Zugangs zu Informationen des Bundes (Informationsfreiheitsgesetz - IFG) ${ }^{12}$ hat einen echten Paradigmenwechsel eingeleitet. ${ }^{13}$ Denn der zuvor geltende Grundsatz der „,beschränkten Aktenöffentlichkeit“14 sah Informationsansprüche nur für diejenigen vor, die als Beteiligte eines Verwaltungsverfahrens die Kenntnis der Akten zur Geltendmachung ihrer subjektiven Rechte benötigten, war also materiellrechtsakzessorisch ausgestaltet. Bürokratisches Arkandenken, das auf Abschottung der Wissensbestände von den Bürgern gerichtet ist, erscheint im Internetzeitalter jedoch nicht nur anachronistisch, sondern offenbart sich auch nur allzu oft als dysfunktional, wenn nämlich Geheimhaltung nicht mehr als Mittel zur Verfolgung von Zielen eingesetzt, sondern zum Selbstzweck wird. Indes ist staatliche Geheimhaltung teilweise auch Voraussetzung für das Funktionieren staatlicher Einrichtungen, wie etwa das Sozial- und das Steuergeheimnis erweisen: Gesetzgeberische Klugheit muss Transparenz- und Geheimhaltungsbelange zum Ausgleich bringen.

\section{B. Der Ausgleich von Transparenz- und Geheimhaltungsinteressen nach dem IFG}

\section{Voraussetzungslosigkeit des Informationsanspruchs}

$\S 1$ Abs. 1 IFG schafft einen voraussetzungslosen Anspruch für jedermann auf Zugang zu amtlichen Informationen der Bundesbehörden. Die Transparenz staatlichen Handelns wird hierbei nicht als Selbstzweck gesehen, sondern vor allem als Instrument bürgerschaftlicher Verwaltungskontrolle. In ihr verwirklicht sich auch der Gedanke demokratischer Teilhabe. ${ }^{15}$ Wenn die Gerichte vielfach die Anspruchsteller als Sachwalter der Allge-

ner, Der geheime Staat. Arkantradition und Informationsfreiheit in Deutschland, 2006.

12 Gesetz v. 5.9.2005, BGB1. I S. 2722.

13 Friedrich Schoch, Das Informationsfreiheitsrecht in der gerichtlichen Praxis, VBlBW 2010, S. 333; Matthias Rossi, Das Informationsfreiheitsrecht in der gerichtlichen Praxis, DVB1. 2010, S. 555.

14 BT-Drs. 7/910, S. 52.

15 BT-Drs. 15/4493, S. 6 nennt zudem die Akzeptanz des Verwaltungshandelns und die effektive Korruptionsbekämpfung als Gesetzesziele; zu den Funktionen s.a. Schoch, Fn. 4, Einl. Rn. 34 ff. 
meinheit bezeichnen, ${ }^{16}$ so rechtfertigt dies allerdings nicht den Umkehrschluss, eigennützige Informationsbegehren hätten keine Berechtigung. Vielmehr folgt aus der Voraussetzungslosigkeit des Informationsanspruchs die Unbeachtlichkeit des Informationsmotivs. ${ }^{17}$ Dass Antragsteller das IFG zum Teil weniger als Instrument der Verwaltungskontrolle nutzen, sondern vor allem zur Aufdeckung des Fehlverhaltens staatlich beaufsichtigter Unternehmen mit dem Ziel der Verfolgung von Schadensersatzansprüchen, ${ }^{18}$ ist deshalb nicht rechtsmissbräuchlich. ${ }^{19}$ Zudem können eigennützige Anträge ebenso wie Informationsbegehren zivilgesellschaftlicher Organisationen und der Presse einen Beitrag zur Verwaltungstransparenz leisten.

\section{Schutz öffentlicher Belange}

Die Crux eines jeden Informationszugangsrechts sind seine Schranken. Das IFG schützt sowohl private als auch öffentliche Geheimhaltungsinteressen, für die nach dem gesetzgeberischen Verteilungsprinzip jeweils das Gebot enger Auslegung gilt. ${ }^{20}$ Die folgenden Ausführungen beschränken sich auf den Schutz öffentlicher Geheimhaltungsbelange, die in einem umfänglichen Katalog in § 3 IFG normiert sind. Der Gesetzgeber hat - anders als insbesondere für den privaten Belang des personenbezogenen Datenschutzes - für den Schutz öffentlicher Belange keine Abwägung mit überwiegenden öffentlichen Interessen an der Offenlegung vorgesehen, son-

16 BVerwGE 130, S. 236, 246; BVerwG NVwZ 2009, S. 1114, 1116.

17 BVerwGE 135, S. 34, 38; VGH Kassel NVwZ 2010, S. 1036, 1038.

$18 \mathrm{Zu}$ Informationsanträgen an die BaFin als Äquivalent einer gesetzlich nicht vorgesehenen pre-trial discovery Elke Gurlit, Informationsfreiheit und die Verschwiegenheitspflichten der BaFin, NZG 2014, S. 1161; in einer weiteren Fallgruppe erfragen Insolvenzverwalter Zahlungseingänge bei gesetzlichen Krankenkassen als ihren potentiellen Anfechtungsgegnern: BVerwG NVwZ 2011, S. 235; OVG Münster NZI 2008, S. 699; dazu Andreas Nitschke, Auskunftsersuchen des Insolvenzverwalters und Informationsfreiheit, DÖV 2014, S. 1049.

19 Allenfalls Schikane- oder Schädigungsabsicht können den Einwand unzulässiger Rechtsausübung mobilisieren. Ein rechtsmissbräuchliches Handeln liegt noch nicht in anwaltlich veranlassten identischen Massenanträgen, VG Berlin, Urt. v. 23.10.2013 - 2 K 294.12 -, Rn. 44 (juris).

20 BT-Drs. 15/4993, S. 9. 
dern ordnet im Umfang des Vorliegens eines Geheimhaltungsgrundes zwingend die Ablehnung eines Informationsgesuchs an. ${ }^{21}$

\section{Bereichsausnahme für Nachrichtendienste}

Nach $\S 3$ Nr. 8 IFG besteht der Informationszugangsanspruch nicht gegenüber den Nachrichtendiensten sowie Bundesbehörden, soweit sie Aufgaben im Sinne des $\S 10 \mathrm{Nr} 3$ Sicherheitsüberprüfungsgesetz wahrnehmen. Hierdurch wurden das Bundesamt für Verfassungsschutz (BfV), der Bundesnachrichtendienst (BND) und der Militärische Abschirmdienst (MAD) mittels einer Bereichsausnahme vom Gesetz ausgenommen. ${ }^{22}$ Sie müssen deshalb auch nicht solche Informationen offenlegen, die keinerlei Sicherheitsrelevanz besitzen. Die Bereichsausnahme soll den Sicherheitsbehörden jeglichen Begründungsaufwand hinsichtlich der Geheimhaltungsbedürftigkeit ersparen. ${ }^{23}$

Die legislative Erwägung überzeugt (mich) nicht. Gegen sie spricht nicht nur die Struktur des Gesetzes, die für die Geheimhaltung als Ausnahme zum Informationszugangsanspruch gerade eine behördliche Begründung verlangt. Vielmehr erscheint die Bereichsausnahme auch in Anbetracht des emanzipatorischen Anliegens des Transparenzprinzips kontraproduktiv. Zwar mag durch die denkbare Offenlegung geheimdienstlichen Fehlverhaltens das bürgerschaftliche Vertrauen in den Nutzen und die Ef-

21 Hierin wird teilweise ein Systembruch gesehen: Einerseits sind durchaus Konstellationen denkbar, in denen sich überwiegende Informationsinteressen über öffentliche Belange hinwegsetzen sollten, dazu Schoch, Fn. 4, Rn. 44 ff.; andererseits ist ein Abwägungsvorbehalt hinsichtlich des Schutzes personenbezogener Angaben wegen des datenschutzrechtlichen Zweckbindungsgebots nicht unproblematisch, dazu Johannes Masing, Transparente Verwaltung - Konturen eines Informationsverwaltungsrechts, VVDStRL 63 (2004), S. 399; krit. im Hinblick auf den Gleichheitssatz Michael Kloepfer/Kai von Lewinski, Das Informationsfreiheitsgesetz des Bundes, DVB1. 2005, S. $1283 \mathrm{f}$.

22 Eine nur aufgabenspezifische Ausnahme folgt aus $\S 10$ Nr.3 SÜG i.V.m. $§ 1$ SÜFV für die Bundespolizei, das Bundeskriminalamt, die Bundeswehr und das Zollkriminalamt.

23 Der Schutz insbesondere nach $\S 3$ Nr. 1 lit. a und c, Nr. 2 IFG wurde nicht als ausreichend angesehen, da er fiskalisches Handeln nicht erfasse und zudem den Sicherheitsbehörden einen Begründungsaufwand auferlege, BT-Drs. 15/4493, S. 12. 
fektivität der Nachrichtendienste weiter erodieren; ${ }^{24}$ hierbei handelt es sich aber gerade um legitime Folgen einer auch der Verwaltungskontrolle dienenden Öffentlichkeit. ${ }^{25}$ Ungleich größer erscheint hingegen die Gefahr weiterer Ansehensverluste durch die informatorische Abschottung geheimdienstlicher Tätigkeit. Die Fetischierung von Geheimnissen bindet nicht nur erhebliche Teile der verfügbaren Arbeitsressourcen der Dienste, sondern kann sich auch zum Folgeschaden fortfressen, indem Gerüchte als funktionales Äquivalent an die Stelle vorenthaltener Informationen treten. ${ }^{26}$

\section{Vorrang spezieller Geheimhaltungsgesetze}

Der Informationsanspruch ist nach $\S 3 \mathrm{Nr} .4$ IFG des Weiteren ausgeschlossen, wenn die Information einer durch Rechtsvorschrift oder durch die Allgemeine Verwaltungsvorschrift zum materiellen und organisatorischen Schutz von Verschlusssachen geregelten Geheimhaltungs- oder Vertraulichkeitspflicht unterliegt. Mit dem legislativen Desiderat, das Geheimnis im Zusammenhang mit dem Spezialgesetz zu schützen, das bereichsspezifisch über Art und Umfang des Geheimnisschutzes entscheidet, ${ }^{27}$ wird die Reichweite des Informationszugangsrechts letztlich in die Disposition des Fachgesetzgebers gestellt. ${ }^{28}$ Der Vorrang spezialgesetzlichen Geheimnisschutzes läuft quer zur Kategorisierung öffentlicher und privater Schutzgüter, und er gilt unabhängig davon, ob der spezialgesetzli-

24 Siehe die parallelen Erwägungen von Jonathan Klaaren, The South African 'Secrecy Act': Democracy Put to the Test, in diesem Band, S. 131, 145 zur kontraproduktiven Wirkung der Offenlegung korruptiver staatlicher Strukturen, die Vertrauen und Normgeltung weiter zerstört.

25 Hierin liegt auch der Unterschied zur präventiven Wirkung strukturellen Nichtwissens wie strafrechtlicher Dunkelziffern, dazu Heinrich Popitz, Über die Präventivwirkung des Nichtwissens, 1980: Das vollständige Wissen um Normabweichungen würde die Geltung des Normen- und des Sanktionssystems ruinieren.

26 Dazu instruktiv Burkhard Sievers, Geheimnis und Geheimhaltung in sozialen Systemen, 1974, S. 73 ff.; zur Geheimhaltung als sozialer Technik immer noch lesenswert Georg Simmel, Das Geheimnis und die geheime Gesellschaft, in: ders., Soziologie, 1908, S. $38 \mathrm{ff}$.

27 BT-Drs. 15/4493, S. 11.

28 Krit. zu § 3 Nr. 4 IFG als bloßer Rezeptionsnorm Schoch, Fn. 4, § 3, Rn. 135. 
che Geheimnisschutz enger oder weiter als derjenige nach dem IFG ist. ${ }^{29}$ Besonderen gesetzlichen Schutz genießen etwa das Steuer-, Sozial- und Statistikgeheimnis. ${ }^{30}$ Da die Nachrichtendienste ohnehin schon durch $\S 3$ Nr. 8 IFG vom Gesetz ausgenommen sind, kommt den für sie geregelten Geheimhaltungstatbeständen keine weitere Bedeutung zu. ${ }^{31}$

Den ebenfalls durch $\S 3$ Nr. 4 IFG vorgesehenen Vorrang der Geheimhaltungspflicht aufgrund einer entsprechenden Einstufung nach der Verschlusssachen-Anweisung des Bundesministeriums des Innern hat hingegen die Rechtsprechung zu Recht begrenzt: Geheimnisschutz wird Dokumenten nicht schon durch eine entsprechende Klassifizierung zuteil; maßgeblich ist vielmehr, dass die Klassifizierung auch den in der Allgemeinen Verwaltungsvorschriften zugrunde gelegten Einstufungskriterien entspricht, was im Streitfall vom Verwaltungsgericht zu überprüfen ist. ${ }^{32}$

\section{Schutz von Sicherheitsbelangen}

Dass nicht eine formale Klassifizierung, sondern die materielle Geheimhaltungsbedürftigkeit maßgeblich ist, kommt auch in den weiteren Vorschriften zum Schutz von Sicherheitsbelangen zum Ausdruck: Die internationalen Beziehungen und die innere und äußere Sicherheit berechtigen die Bundesbehörden nur insoweit die Geheimhaltung, als das Bekanntwerden der begehrten Informationen nachteilige Auswirkungen auf diese Schutzgüter haben kann ( 33 Nr. 1 lit. a und c IFG), und nur eine durch Offenbarung drohende Gefährdung der öffentlichen Sicherheit befugt die Behörden zur Geheimhaltung ( 3 Nr. 2 IFG). Die Rechtsprechung legt für eine Gefährdung des Schutzguts der öffentlichen Sicherheit polizeirechtlich inspirierte Maßstäbe an, indem sie den Behörden eine faktenbasierte Darlegung auferlegt, weshalb Belange der öffentlichen Sicherheit tatsäch-

$29 \S 9 \mathrm{KWG}, \S 8 \mathrm{WpHG}$ als spezialgesetzliche Verschwiegenheitspflichten der BaFin schützen in erster Linie die Betriebs- und Geschäftsgeheimnisse der beaufsichtigten Unternehmen, bleiben aber im Schutzumfang hinter § 6 IFG zurück, dazu Gurlit, Fn. 18, S. $1164 \mathrm{f}$.

$30 \S 30$ AO, $\S 35$ SGB I, § 16 BStatG; zum Statistikgeheimnis s. VG Wiesbaden GewArch 2013, S. 255.

31 Anders wohl BT-Drs. 15/4493, S. 11; als nachrichtendienstliche Geheimhaltungsregeln kommen im Übrigen allein die Beschränkung von Auskunftsansprüchen nach $\S 15$ Abs. 2 BVerfSchG, § 9 BNDG und $\S 7$ MADG in Betracht.

32 BVerwG NVwZ 2010, S. 326; NVwZ 2010, S. 321, 324 ff. 
lich durch das Bekanntwerden von Informationen gefährdet werden. ${ }^{33}$ Durch diesen Maßstab wird sichergestellt, dass die Geheimhaltung von Informationen begründungsbedürftige Ausnahme ist.

Wesentlich großzügiger bestimmt hingegen das Bundesverwaltungsgericht den Schutz internationaler Beziehungen. Im Streit um die Offenlegung von Flugbewegungen, die Aufschluss geben konnten über vermutete - und inzwischen längst bestätigte ${ }^{34}$ - Gefangenentransportflüge der USamerikanischen CIA in Deutschland, billigte das Gericht der Bundesregierung nicht nur einen Beurteilungsspielraum für die Gestaltung und Ziele der auswärtigen Beziehungen zu, sondern erstreckte diesen darüber hinaus auf die Annahme der nachteiligen Auswirkungen einer Offenlegung: Deren Prognose - gemessen am diplomatischen Ziel der Vermeidung weiterer „Verstimmungen“ des Bündnispartners - bedürfe nicht der Darlegung von Tatsachen, sondern könne auf Einschätzungen gestützt werden, die ihrerseits gerichtlich nicht überprüfbar sind. ${ }^{35}$ Mit dieser Anerkennung eines gerichtlich kontrollfreien Raums dürfte angesichts der zahlreichen Felder internationaler Kooperation der Regierung ein veritabler Geheimhaltungsgrund zugewachsen sein. ${ }^{36}$

\section{Schutz des behördlichen Entscheidungsbildungsprozesses}

$\S 3$ Nr. 3 lit. b IFG schützt die vertraulichen Beratungen von Behörden, § 4 IFG sieht als Sollvorschrift die Ablehnung von Informationsanträgen für Entwürfe und vorbereitende Dokumente vor, soweit und solange durch die

33 OVG Koblenz NVwZ 2008, S. 1141, 1143: Angabe über Unternehmen, die der Störfall-Verordnung unterliegen, darf nicht pauschal unter Hinweis auf die Gefahr terroristischer Anschläge abgelehnt werden; zur Maßgeblichkeit der polizeirechtlichen Maßstäbe auch BVerwG NVwZ 2011, S. 1072, 1073; in gleichem Sinne verfährt die Rechtsprechung bei der Anwendung von $\$ 3$ Nr. 1 lit. d IFG, s. VGH Kassel NVwZ 2010, S. 1036, 1039 f.; s.a. BVerwG NVwZ 2012, S. 112, 114 f. im Rahmen von $\S 99$ Abs. 1 S. 2 VwGO.

34 Abschlussbericht des Untersuchungsausschusses vom 31.3.2009, BT-Drs. 16/13400, S. 397, 481.

35 BVerwG NVwZ 2010, S. 321.

36 Zu Recht krit. Matthias Goldmann, Urteilsanmerkung, JZ 2010, S. 572; für die Ablehnung des Zugangs zum Terminkalender der Bundeskanzlerin wurde ein Beurteilungsspielraum hinsichtlich nachteiliger Auswirkungen auf die innere und äußere Sicherheit i.S.v. § 3 Nr. 1 lit. c IFG anerkannt, OVG Bln-Bbg NVwZ 2012, S. 1196, 1199. 
vorzeitige Bekanntgabe der Erfolg der behördlichen Maßnahme vereitelt würde. Die prozedural ausgerichteten Geheimhaltungsgründe ${ }^{37}$ lassen sich nur schwer voneinander abgrenzen. $\S 3 \mathrm{Nr} .3$ lit. b IFG will in erster Linie den unbefangenen und freien zwischen- und innerbehördlichen Meinungsaustausch schützen, während §4 IFG an die externen Wirkungen einer frühzeitigen Offenbarung anknüpft. Die Vorschriften unterscheiden sich zudem in der zeitlichen Erstreckung des Schutzes. Kann die notwendige Vertraulichkeit von behördlichen Beratungen möglicherweise auch dann Schaden nehmen, wenn die Beteiligten befürchten müssen, dass nach Verfahrensabschluss Informationen offenbart werden, so ist der Schutz nach $\S 4$ IFG von vornherein auf laufende Verwaltungsverfahren beschränkt. ${ }^{38}$ Die von den Gerichten für $\S 3$ Nr. 3 lit. b IFG vorgenommene Differenzierung zwischen geschützten entscheidungsvorbereitenden Dokumenten und solchen Informationen, die als Tatsachenmaterial offenzulegende Grundlage der Entscheidung sind, ${ }^{39}$ wird sodann im Wortlaut des $\S 4$ Abs. 1 S. 2 IFG für laufende Entscheidungsverfahren aufgegriffen. Im Ergebnis stellen die Gerichte hohe Anforderungen an die Berufung auf die Vertraulichkeit des innerbehördlichen Entscheidungsbildungsprozesses.

\section{Unausforschbarer Kernbereich der Regierung}

Der vom Bundesverfassungsgericht geschöpfte Kernbereich exekutiver Eigenverantwortung, der einen nicht ausforschbaren Initiativ-, Beratungsund Handlungsbereich der Regierung umfasst, ist zwar im IFG nicht als Geheimhaltungsgrund geregelt, wird aber als ungeschriebener verfassungsunmittelbarer Schutz auch dem Informationsanspruch entgegengehalten. ${ }^{40} \mathrm{Da}$ sich das Regierungshandeln aber im Rahmen des IFG als behördliche Aufgabenwahrnehmung darstellt, ist es nicht per se dem Anwendungsbereich des Gesetzes entzogen. ${ }^{41}$ Vielmehr gilt hier wie auch bei der Verwaltungstätigkeit im Übrigen, dass es der Begründung bedarf, wes-

37 BVerwG NVwZ 2010, S. 194, 195; BVerwG NVwZ 2010, S. 1495, 1496.

38 BVerwG NVwZ 2011, S. 1072; BVerwG ZUR 2012, S. 183, 186; Schoch, Fn. 4, $\S 3$ Rn. 130, § 4 Rn. $31 \mathrm{f}$.

39 OVG Münster NWVB1. 2014, S. 268, 270; VG Berlin JZ 2012, S. 796, 797.

40 BT-Drs. 15/4493, S. 12; BVerwG ZUR 2012, S. 183, 187; VG Berlin JZ 2012, S. 796, $797 \mathrm{f}$.

41 BVerwG ZUR 2012, S. 183, 184 ff.; BVerwG NVwZ 2012, S. 251, 254 - Akten des Bundesministeriums der Justiz sind vom IFG erfasst. 
halb die Offenlegung zu einer Störung des inneren Entscheidungsbildungsprozesses der Regierung führt. ${ }^{42}$ Hierbei ist die Annahme eines absolut geschützten Kernbereichs bei ministeriellen Vorbereitungsarbeiten zu Gesetzen eher fernliegend. ${ }^{43}$ Mit der Durchsetzung der Transparenz auch des Regierungshandelns haben die Gerichte dem IFG einen Bereich erschlossen, der insbesondere für die demokratische Teilhabe der Gesellschaft von erheblicher Bedeutung ist. ${ }^{44}$

\section{Zur Wirksamkeit des Informationszugangsrechts}

Wurde mit dem IFG das Transparenzprinzip durchgesetzt und staatliche Geheimhaltung zur begründungsbedürftigen Ausnahme? Der umfängliche Katalog von Ausnahmen zum Schutz öffentlicher Belange lässt daran zweifeln. Er ist Folge der Durchsetzung von Ressortegoismen bei der interministeriellen Gesetzesvorbereitung und gilt als „Verlustliste der Informationsfreiheit". 45

Gleichwohl zeigt sich, dass das Informationsfreiheitsgesetz inzwischen im Alltag der Behörden angekommen ist: Wurden im Jahr 2006 nur knapp 2.300 Informationsanträge an die Bundesbehörden gerichtet, so ist diese Zahl im Jahr 2014 auf nahezu 8.700 Anträge angewachsen. ${ }^{46}$ Informationsverweigerungen der Behörden sorgten dafür, dass das vergleichsweise schmale Gesetz auch eine steile gerichtliche Karriere gemacht hat. Als pathologische Spitze vermitteln gerichtliche Entscheidungen zwar kein verlässliches Bild der Struktur der Nutzer des Gesetzes und der Natur der von ihnen begehrten Informationen. Sie zeigen aber, dass das Gesetz durchaus

42 BVerwG ZUR 2012, S. 183, 187 bezweifelt, dass es in Anbetracht von $\S 3$ Nr. 3 lit. b IFG des Rückgriffs auf die ungeschriebene Figur des Kernbereichs bedarf; ähnlich VG Berlin JZ 2012, S. 796, 797; s.a. Schoch, Fn. 4, Vorb. §§ 3-6, Rn. 27.

43 BVerwG ZUR 2012, S. 183, 187 sah im ministeriellen Vorbringen „das Bild einer Ministerialverwaltung mit einem eher geringen Selbstbewusstsein".

44 Benedikt Assenbrunner, Bürgerbeteiligung bei der Kontrolle der Ausübung von Regierungsgewalt, DÖV 2012, S. 547; s.a. Hans-Peter Roth, Regierungstätigkeiten im Lichte der Informationsfreiheit, DÖV 2012, S. 717.

45 Kloepfer/von Lewinski, Fn. 21, S. 1280; Schoch, Fn. 4, Vorb. § 3, Rn. 7 ff.

46 Zum Antragsaufkommen aufgeschlüsselt nach Ministerien und Behörden s. IFGStatistik des Bundesministeriums des Innern, abrufbar unter http://www.bmi.de. Hinzu treten Informationsanträge nach dem Verbraucherinformationsgesetz (VIG) und den Umweltinformationsgesetzen von Bund und Ländern. 
auch von den Medien und zivilgesellschaftlichen Organisationen genutzt wird $^{47}$ und damit von Interessenten, die als Multiplikatoren die Verwaltungstransparenz verstärken.

Die Zahl verwaltungsgerichtlicher Judikate ist allerdings auch Beleg dafür, dass der Informationszugang immer noch teilweise gegen eine Verwaltungskultur durchgesetzt werden muss, die den interessierten oder auch nur neugierigen Bürger als Störenfried wahrnimmt, der die Verwaltung von der Erfüllung ihrer eigentlichen Aufgaben abhält. Die Verwaltungsgerichte verstehen sich hierbei aber als Sachwalter der Öffentlichkeit und folgen ganz überwiegend dem Grundsatz, dass Transparenz die Regel, Geheimhaltung hingegen die Ausnahme sein soll. ${ }^{48}$

\section{Informationszugangsrechte als Element einer Informationsrechtsordnung}

Wenn das Informationsfreiheitsgesetz Verwaltungstransparenz und legitime Geheimhaltungsinteressen zum Ausgleich bringen muss, so ist dies Ausdruck der Tatsache, dass verfassungsrechtlich das eine nicht ohne das andere zu haben ist. ${ }^{49}$ Das IFG ist damit Element einer Informationsrechtsordnung im weiteren Sinne, die nicht nur die Regeln für die Verteilung von Informationen, sondern auch für ihre Erzeugung und für ihre weitere Verarbeitung bereithält. Gegenstand einer Informationsrechtsordnung sind dabei nicht nur staatlich geschöpfte Informationen, sondern auch privat verwaltete Wissensbestände, wie etwa diejenigen der Informationsgiganten Facebook, Google und Co. Derartige Regeln muss nicht nur

$47 \mathrm{Zu}$ Informationsanträgen von Journalisten s. nur beispielhaft BVerwG NVwZ 2010, S. 321: Antrag betr. Aktivitäten der CIA in Deutschland; OVG Bln-Bbg NVwZ 2012, S. 1196: Antrag betr. Einladungsliste der Bundeskanzlerin zu ihrer Geburtstagsfeier; OVG Bln-Bbg K\&R 2014, S. 141: Antrag betr. Rechtsgutachten des wissenschaftlichen Dienstes des Bundestages; s.a. die empirische Untersuchung von Jan Ziekow/Alfred Debus/Elisabeth Musch, Bewährung und Fortentwicklung des Informationsfreiheitsrechts, 2013.

48 Elke Gurlit, Das Informationsverwaltungsrecht im Spiegel der Rechtsprechung, Die Verwaltung 44 (2011), S. $102 \mathrm{f}$.

49 Jonathan Klaaren, The South African 'Secrecy Act': Democracy Put to the Test, in diesem Band, S. 131, 152, kleidet die Wechselbezüglichkeit von Transparenz und Geheimhaltung in die Metapher des entanglement politischer Werte. 
das Verwaltungsrecht bereitstellen. ${ }^{50}$ Vielmehr leisten auch die weiteren Teile der Rechtsordnung in Ausfüllung verfassungsrechtlicher Vorgaben ihren jeweiligen Beitrag. Wesentliche Impulse setzt überdies das Europarecht.

Rechtspolitisch ist hierbei der Trend zu mehr staatlicher Transparenz nicht mehr aufzuhalten. Hierfür steht die innovative open data-Gesetzgebung einiger Bundesländer, die den Bürger nicht mehr auf Informationsgesuche verweist, sondern aktiv ihre Wissensbestände über Internetportale zugänglich macht und die Interessenten zur Nutzung und Weiterverarbeitung auch von raw data im Interesse des Gemeinwohls anhält. ${ }^{51}$ Verteilungsregeln findet sich indes nicht nur in Informationszugangsgesetzen, sondern auch im Datenschutzrecht. Das Grundrecht auf informationelle Selbstbestimmung ist dabei nicht allein ein Instrument zur Abschottung von Informationen, sondern umfasst gerade auch das Recht zu wissen, wer was über einen weiß. ${ }^{52}$ Muss die Informationsrechtsordnung insbesondere auch der Erkenntnis Rechnung tragen, dass Gefährdungen individueller Freiheit nicht nur von staatlichen Sicherheitsbehörden ausgehen, sondern auch von Facebook, Google und Co, so stellen sich informationsrechtliche Anforderungen an das die privaten Rechtsbeziehungen leitende Zivilrecht. ${ }^{53}$ Ein neuerer Trend ist etwa, das privatrechtliche Wettbewerbsrecht zur Sanktionierung von Datenschutzverstößen von Unternehmen einzusetzen. ${ }^{54}$ So können nunmehr Verbraucherschutzverbände mit Unterlassungs-

$50 \mathrm{Zu}$ einem Informationsverwaltungsrecht Rainer Pitschas, in: Wolfgang HoffmannRiem/Eberhard Schmidt-Aßmann/Gunnar Folke Schuppert (Hrsg.), Reform des allgemeinen Verwaltungsrechts, 1993, S. 219; s.a. Masing, Fn. 21, S. 432; Friedrich Schoch, Öffentlich-rechtliche Rahmenbedingungen einer Informationsordnung, VVDStRL 57 (1998), S. 158.

51 Christoph Schnabel, Das neue Hamburgische Transparenzgesetz, NordÖR 2012, S. 431; in Rheinland-Pfalz wurde am 27. November 2015 das Landestransparenzgesetz beschlossen.

52 BVerfGE 65, S. 1, 43.

53 Verfassungsrechtlicher Hebel ist hierbei die staatliche grundrechtliche Schutzpflicht, die in den Rechtsverhältnissen Privater zum Tragen kommen muss, dazu BVerfGE 84, S. 192, 195; BVerfGE 89, S. 214; zum verfassungsgebotenen Datenschutz im privaten Versicherungsrecht BVerfGK 9, S. 353, 358 ff.; BVerfGK NJW 2013, S. 3086; s.a. § 213 VVG; zu den unionalen Anforderungen nach Art. 7, 8 GRCh EuGH NVwZ 2014, S. 857 - González; zuvor EuGH EuZW 2010, S. 939 Schecke.

$54 \mathrm{Zu}$ Datenschutzvorschriften als Marktverhaltensregeln i.S.v. § 4 Nr. 11 UWG s. OLG Karlsruhe NJW 2012, S. 3312. 
klagen unternehmerische Datenschutzverstöße unterbinden. ${ }^{55}$ Sowohl für die Erzeugung als auch für die Nutzung von Informationen leisten überdies die Instrumente des gewerblichen Rechtsschutzes wie das Urheberund Patentrecht einen Beitrag. ${ }^{56}$ Schließlich kann auch das Strafrecht Regeln für die Zulässigkeit von Informationsverarbeitungen und -verteilungen bereithalten.

In der Fortentwicklung einer konsistenten Informationsrechtsordnung liegt ein anspruchsvolles Arbeitsprogramm für Gesetzgebung, Wissenschaft und Praxis. Packen wir es an!

55 Gesetz zur Verbesserung der zivilrechtlichen Durchsetzung von verbraucherschützenden Vorschriften des Datenschutzes vom 7. Februar 2016, BGBl. I S. 233.

56 Erzeugung und Verteilung wird verknüpft in dem neuen urheberrechtlichen Instrument des open access nach $\S 38$ Abs. 4 UrhG, das den wissenschaftlichen Urhebern ein Zweitveröffentlichungsrecht einräumt. 